\title{
What are patients' care experience priorities?
}

\author{
Rick A ledema, ${ }^{1}$ Blake Angell ${ }^{2}$
}

${ }^{1}$ Faculty of Health, The University of Tasmania, Sydney, New South Wales, Australia

${ }^{2}$ The George Institute for Global Health, University of Sydney, New South Wales, Australia

\section{Correspondence to} Professor Rick A ledema, Faculty of Health, The University of Tasmania, Sydney, New South Wales, Australia;

Rick.iedema@utas.edu.au

Accepted 14 April 2015 http://dx.doi.org/10.1136/ bmjqs-2014-003691
We are investing considerable resources in defining and measuring patients' care expectations. Such measurement will yield insight into whether and how services are meeting patients' experience expectations. But because measurement is inherently distanced in time and space, it does not resolve patients' experience of feeling 'reluctant to directly challenge healthcare professionals ${ }^{1}$ about issues that matter to them now and which may make them feel unsafe.

Research has shown that when patients and family members experience concerns about their care, they want to be able to discuss these experiences with their clinicians. They often want such discussions to include explanations from professionals and dialogue about 'what happened' and about tensions, uncertainties and contradictions. ${ }^{2}$

Patients and family members interviewed for a large incident disclosure study reported that they appreciated that care is complex, ${ }^{3}$ and that there may be no simple answers to explain care problems. ${ }^{4}$ But being granted the time for dialogue with their clinicians and service representatives reassured patients and family members that their concerns and questions were taken seriously. This meant for them in turn that similar events might be prevented from happening again. They also felt that dialogue reassured them that their views on and advice about how to improve care were respected. When patients have concerns about their care, the timing and authenticity of such dialogue are seen as paramount.

For its part, measuring patients' experiences presupposes abstracting 'what happened to you' into a general metric that applies to 'people like you'. Measurement is, in the first instance, about the service and the system. Dialogue is about the patient and their family. Measurement will never obviate patients' providing feedback about their concerns to their own clinicians and their local services.
What started out as satisfaction surveying in the $1970 \mathrm{~s}^{5}$ has now morphed into a major industry, with Picker, ${ }^{6}$ Press Ganey ${ }^{7}$ and HCAHPS $^{8}$ (the US-based 'health consumer assessment of healthcare providers and systems') leading the way. A number of landmark government reports has now enshrined the role of patient feedback measurement in healthcare governance and practice improvement. ${ }^{9}{ }^{10}$ In addition to the hundreds of 'patient reported outcome measures"11 that have to date been developed to measure whether care meets patients' care-outcome expectations, 'patient reported experience measures' (PREMs) ${ }^{12}$ invite feedback about service features such as waiting times, admission processes, staff responsiveness, discharge processes and so forth. PREMs also elicit feedback on matters ranging from clinical team communication to service responsiveness to patient needs, accessibility of information, environmental factors (lighting, space design), equipment availability and functionality, and they have been shown to shed light on clinical outcomes. ${ }^{13} 14$

It is this rapidly growing area of 'patient experience' research that is the focus of the article by Rebecca Lawton and colleagues. ${ }^{15}$ Following this team's development of an overview of factors contributing to patient safety incidents (referred to as the Yorkshire Contributory Factors Framework $\left.(\mathrm{YCFF})^{16}\right)$, patient measure of safety (PMOS) was constructed from an analysis of the priorities attributed by 33 patient interviews to YCFF. ${ }^{1}$ The team's most recent article reports on a comparison of PMOS against a range of other safety rating instruments.

For the purpose of making such comparisons possible, data were collected from 33 hospital wards across three acute hospitals, including responses from staff for the four outcome measures in the Hospital Survey of Patient Safety Culture, patient responses to the PMOS and the so-called Friends and 
Family Test ('Would you recommend this service to a friend or family member?'). The study also collated publicly reported safety outcome data for 'harm-free care' on each ward. The article's main finding is that PMOS provides a perspective on safety and improvement that both confirms and complements information produced from other safety measures.

What PMOS adds to our understanding of safety is that it creates an intersection between patient safety factors and patients' observations of care going on around them. Like PREMs and HCAHPS (measures that were not included in the study's comparison it should be noted), PMOS asks patients' judgements about matters relating to their own care. Casting its feedback net a little wider than PREMs and HCAHPS, PMOS opens the door for patients to evaluate care from a rather more general perspective. For example, patient ratings of clinical team functioning and organisational culture are invited with survey statements like: 'staff did not work together as a team here', 'staff seemed to struggle to help when they needed it' and 'nurses were always able to get help from other staff'.

In eliciting patients' views on what they see going on around them, PMOS invests faith in patients' ability to provide 'eyewitness' accounts. This is not surprising. A number of studies have now reported patients to be keen to provide their local service with advice, particularly when care goes wrong: "What I [patient] had started to do [during my care] was to write down a journal and do an evaluation of what was going on in the hospital and where the problems were." 4 These studies also make clear that patients' feedback may harbour critical implications for service improvement. ${ }^{17}$

Of course, patients may get it wrong. ${ }^{18}$ It is striking, though, that more and more healthcare service improvement initiatives involve patients and draw on their experiences to inspire and guide change. ${ }^{19}$ It is patients' lived experiences in particular that play an increasingly important role at conferences and meetings. For patients, the next best thing to here-and-now dialogue is indeed post hoc story-telling. Stories can recount what happened in all its complexity, including tensions, uncertainties and contradictions. Stories are good for enabling us to make sense of confusing experiences and complex circumstances: ${ }^{20}$ something can make sense even when we lack answers and certainty.

We make these observations by way of background to three questions we want to pose about Lawton and colleagues' study. ${ }^{15}$ Our first question concerns the measurability of non-standard experiences, the second relates to the translation of measurement into practice change and the third asks about the governance approach and service responses favoured by patients when their care falls short.
PMOS was originally designed to accommodate non-standard care experiences from data obtained from 14 non-Caucasian/British interviewees and 19 white/British interviewees. ${ }^{1}$ The inclusion of patients from culturally and linguistically diverse backgrounds is significant as they are more likely to experience substandard care, incidents and less than ideal treatment trajectories. ${ }^{21}$ Lawton and colleagues' present study draws on the input from predominantly white British patient respondents. The question that arises here then is whether the inclusion of patients from less well-integrated and more recent immigrant populations in the design of PMOS might have yielded different items, and whether their inclusion in the comparison between PMOS and other safety measures might have yielded different results.

The second question is about how we convert PMOS measurement results and rating comparisons into practice. If results highlight persistent problems in a particular domain of care or a particular service-say, a lack of respect for patient's need of privacy-that domain or service may be targeted with a tailored improvement programme. Patients' interview statements that were originally used as the basis for PMOS domains are quite revealing about how to design such a programme: "This one lady she has had a line on her chest ... and a few times they have not put the curtain around ... her." ${ }^{13}$ Designing a programme without reference to specific local problems will render it abstract and decontextualised, and its purpose and focus may not be apparent to those targeted. Learning is most effective when tied to specific experiential circumstances, because behaviour is not necessarily fully conscious, ${ }^{22}$ and the learner needs to recognise herself and her behaviour in what is learned. Abstract formal learning remains at too many removes from actors' practical awareness for it to translate into behaviour change. As pedagogical theory has taught us, we need to create opportunities for learners to connect learning to actual in situ behaviour and personally experienced situations for them to be able to close the loop between information, knowledge, selfidentity and practice. ${ }^{23}{ }^{24}$ Measurement may be promoted as service improvement resource, but its distance in time and space from in situ care and local behaviour considerably limit its effectiveness for achieving change for patients.

Our third question rests on the finding that patients experience 'reluctance to directly challenge healthcare professionals'. In practice, studies have found that patients' feedback to their own care providers is not systematically encouraged, welcomed or used for practice improvement. ${ }^{4}$ When care is not to patients' satisfaction, let alone when care goes wrong, discussions with clinicians and service representatives about concerns and problems are more challenging and less rewarding than patients expect them to be. ${ }^{25}$ At the same time, patients are exhorted to become proactive about care safety. ${ }^{26}$ Yet, it is evident that patients 
already play a credible and important, albeit invisible, role in keeping their own and others' care safe. ${ }^{27}$

Let us not forget that patients are among the first to experience the baffling complexity of contemporary care. Just think of the times we have been a patient and were told: "Ms Smith, you need to be across the road for that test. And this afternoon you're not seeing Dr Jones because she is on leave, and instead you'll be seeing Dr Bloggs whose office is on level 9." What to insiders may appear to be everyday occurrences are for patients bafflingly complex scenarios. No doubt, this is not unrelated to patients being more and more likely to present with multiple problems. Patients also interact with increasingly specialised practitioners and services experiencing high levels of staff turnover. Practitioners often experience uncertainty about treatments and resource decisions, as well as having to accommodate ongoing technological change, scientific development and organisational reform. When asked about their care experiences, patients often talk about complex care journeys, variable impressions and persistent questions. $^{25}$

It is also clear that chronically ill patients, alongside culturally and linguistically diverse background patients, are particularly at risk in this regard. ${ }^{21}$ Their journeys and experiences are complex, unique and often fraught. While these 'complex patients' may be increasingly targeted with trials, surveys and interviews, they do not always feel that such studies make it possible for them to relate issues that matter to them. ${ }^{3}$ When they feel unsafe, patients should be able to provide direct feedback and see that feedback is translated into action when and where they receive their care. At the least, they should be able to expect that their fellow patients be spared the problems that they happened to witness or personally experience. ${ }^{4}$

These expectations raise the bar in two ways. First, to ensure that-besides making possible interservice ratings and comparisons-patients' experiences produce meaningful and timely impact on care as practice, healthcare services need to adopt a proactive stance towards patients' in situ feedback. This is because patients' experiences-whether scientifically measured or told as post hoc stories-continue to have a tenuous link with service improvement. ${ }^{27}$ Second, to ensure that the generic and abstract aspects of patients' experiences and their richness and diversity become integral to practice improvement, we need to grant patients a greater say in how they provide feedback, and how their feedback is converted into practice. In spite of all the effort put into patientfocused research, it is still the case that patients 'are rarely given the opportunity to represent themselves in ways that enable them to highlight their own points of view and issues of concern, or to provide creative insights that they themselves hold dear'. (personal communication, Collier A and Wyer M, 2015).
Competing interests None declared.

Provenance and peer review Not commissioned; internally peer reviewed.

\section{REFERENCES}

1 Giles SJ, Lawton RJ, Din I, et al. Developing a patient measure of safety (PMOS). BMJ Qual Saf 2013;22:554-62.

2 Iedema R, Allen S, Britton K, et al. Patients' and family members' views on how clinicians enact and how they should enact incident disclosure: the ' 100 patient stories' qualitative study. BMJ 2011;343:d4423.

3 Iedema R, Allen S. Anatomy of an incident disclosure: the importance of dialogue. Jt Comm J Qual Patient Saf 2012;38:435-42.

4 Iedema R, Allen S, Britton K, et al. What do patients and relatives know about problems and failures in care? BMJ Qual Saf 2012;21:198-205.

5 Williams B. Patient satisfaction: a valid concept? Soc Sci Med 1994;38:509-16.

6 Jenkinson C, Coulter A, Bruster S. The Picker Patient Experience Questionnaire: development and validation using data from in-patient surveys in five countries. Int J Qual Health Care 2002;14:353-8.

7 Press Ganey. Clients that switched from in-house surveying to Press Ganey. South Bend, IN: Press Ganey, 2010.

8 Centers for Medicare \& Medicaid Services. HCAHPS fact sheet. Baltimore, MD: Centers for Medicare \& Medicaid Services, 2013.

9 Darzi A. High quality care for all. London: Stationery Office, 2008.

10 Department of Health. NHS Outcomes Framework 2012/13. London: NHS, 2011.

11 Nelson EC, Eftimovska E, Lind C, et al. Patient reported outcome measures in practice. BMJ 2015;350:g7818.

12 de Silva D. Measuring patient experience. London: The Health Foundation, 2013.

13 Varagunam M, Hutchings A, Black N. Do patient-reported outcomes offer a more sensitive method for comparing the outcomes of consultants than mortality? A multilevel analysis of routine data. BMJ Qual Saf 2015;24:195-202.

14 Black N, Varaganum M, Hutchings A. Relationship between patient reported experience (PREMs) and patient reported outcomes (PROMs) in elective surgery. BMJ Qual Saf 2014;23:534-42.

15 Lawton R, O'Hara JK, Sheard L, et al Staff and patient perspectives on hospital safety: are they different and do they predict harm free care? BMJ Qual Saf 2015:1-8.

16 Lawton R, McEachan RR, Giles SJ, et al. Development of an evidence-based framework of factors contributing to patients safety incidents in hospital settings: a systematic review. BMJ Qual Saf 2012;21:369-80.

17 Weissman JS, Schneider EC, Weingart SN, et al. Comparing patient-reported hospital adverse events with medical record review: do patients know something that hospitals do not? Ann Intern Med 2008;149:100-8.

18 Buetow S, Kiata L, Liew T, et al. Patient error: a preliminary taxonomy. Ann Fam Med 2009;7:242-31.

19 Quaid D, Thao J, Denham CR. Story power: the secret weapon. J Patient Saf 2010;6:5-14.

20 Boyd B. On the origin of stories: evolution, cognition and fiction. Cambridge, MA: Harvard University Press, 2009.

21 Smedley BD, Stith AY, Nelson AR. Unequal treatment: confronting racial and ethnic disparities in health care 
(Committee on Understanding and Eliminating Racial and Ethnic Disparities in Health Care, Board on Health Sciences Policy). Washington DC: Institute of Medicine-National Academy Press, 2003.

22 Sax H, Clack L. Mental models: a basic concept for human factors design in infection prevention. J Hosp Infect 2015;9:335-9.

23 Kolb D. Experiential learning: experience as the source of learning and development. EnglewoodCliffs, NJ: Prentice Hall, 1984.

24 Boud D, Miller N. Working with experience: animating learning. London: Routledge, 1996.
25 Iedema R, Sorensen R, Manias E, et al. Patients' and family members' experiences of open disclosure following adverse events. Int J Qual Health Care 2008;20: 421-32.

26 Davis R, Koutantji M, Vincent C. Patients' willingness to question health care staff on issues related to their medical treatment. Qual Saf Health Care 2015; 17:90-6.

27 Hor S, Godbold N, Collier A, et al. Finding the patient in patient safety. Health (London) 2013;17:567-83. 